\title{
Design of Standardized Data Process in 4PL Freight Management
}

\author{
Yan Zhang ${ }^{1, \text { a }}$, Qiong Sun ${ }^{2, b}$ \\ ${ }^{1}$ Department of Science and Technology, Shenyang University, Shenyang, 110044, China \\ ${ }^{2}$ Department of Foreign Languages, Shenyang University, Shenyang, 110044, China \\ aemail: sdxinxi@126.com, bemail:sunqiong668@163.com
}

Keywords: 4PL; TPL; standardized process; horizontal supply chain

\begin{abstract}
The problems of low informatization and low intensification are present in China's TPL industry, and the key way to improve logistics level is to imrove the informatiztion level of TPL. In view of the situation that current management of 4PLplatform can't meet the practical application needs of TPL enterprises, this paper proposes the building of such a 4PL platform that is based on the real needs of most TPL enterprises, serves most TPL, ensures that the management of every TPL enterprise and branch fits the current reality, and at the same time, builds up horizontal supply chain so that TPL enterprises can share warehousing resources, freight resources and joint distribution.
\end{abstract}

\section{Introduction}

Every 1\% cost reduction in China's logistics will bring about 100 billion of social assets, and China's logistics still has $6 \%-10 \%$ of improvement room compared with that of developed nations ${ }^{[1]}$. The key way to improve China's logistics is to improve TPL's informationization. The size of TPL enterprises in China is relatively small, according to materials, there are more than 700,000 domestic registered TPL enterprises ${ }^{[2-4]}$, but the number of enterprises in actual operation reaches 10 million, that is to say, the proportion of informationization is less than $10 \%^{[4]}$. Based on this, our country stresses strengthening logistics informationization in the $11^{\text {th }}$ and $12^{\text {th }}$ five-year plan. On Jan. 7, 2013, the Department of Industry and Informationization issued the Guidelines of Improving Logistics Informationization ${ }^{[5]}$, putting forth suggestions to fasten the building of a standardized system for logistics informationization.

To solve the prevailing problems of low informationization and low intensification in medium and small TPL enterprises, many experts have proposed that building 4PLplatform and using 4PL facing supply chain management can reduce traction costs, commodity transferring costs and logistical process, bring tremendous social cost reduction and profit increase ${ }^{[6]}$. This view has reaped common understanding in this industry and at present many researchers, including the government, scholars, software designers and institutions, are all committed to the study and design of $4 \mathrm{PL}{ }^{[7]}$. The ideal is condition is that all the supplying and purchasing activities of clients are managed by 4PL. But in practice, the current 4PLplatform suffers from the flaw that its process does not fir TPL reality, which makes TPL informatization the bottleneck of the industry.

The author proposes building such a 4PL management platform that, with the building of a standardized process, serves a number of TPL, establishes horizontal supply chain among TPL, ensures the management of each TPL's branches fit the business reality and at the same time ensures that TPL enterprises and their branches can from cloud logistics on the Web, so that TPL enterprises can manage themselves through the platform with the lowest cost and TPL enterprises can share warehousing resources, freight resources and joint distribution, forming a business mode unique to TPL enterprises ${ }^{[8-9]}$. A standardized process of freight management in 4PL platform building is designed, including door to door service, waiting for reception, inbound, outbound, outbound list, standard outbound procedures, meanwhile, the component computation algorithm of net revenue, receivables, inventory temporary charges, alternative payments, fund balances and cash balances is provided. 


\section{to prepare cargo}

There are two ways for cargo to be transported from shipping enterprises to TPL. One is known as waiting for delivery, that is, shipping enterprises send the goods to TPL. The other is that TPL take delivery of cargo from shipping enterprises (door to door service).

\section{A. Door to door service}

The management procedures of door to door service are shown in Figure1. The people who take delivery of cargo from shipping enterprises are usually delivery staff, who take delivery, accept the orders and cargo, and fill out cargo receipts for shipping enterprises. The cargo receipts should contain the following: (1) information of the destination (destination, name of the store); (2) date of cargo reception, cargo number; (3) information of the shipping enterprise (name, number, and telephone number of the shipping enterprise, consignee's name and telephone number); (4) information of charge types (rates, types of charges); (5) types of charges (cash, account period, the arrival pay, back pay, own delivery, other charges, total); (6)information of the cargo (the order numbers, packages, number of packages, volume, weight, amount, remarks); (7) simplified shipping agreement: specification of cargo, insurance, package, scope of coverage, terms of cargo loss and damage compensation claims, terms of delivery and claims; (8) other information (declared value, alternative payments, number of orders, number of inventory documents).

The current informationization situation of this receipt can’t connect 4PL to each shipping enterprise, the problem being not technology but cost. The principle of low cost must followed ${ }^{[10]}$. In this platform this receipt is still designed to be hand-written in printed documents, so that each undertaking company can afford the application cost of this platform. Cargo receipts are in triplicate. After the consignor signs, the consignor keep one of the receipts, delivery staff takes to orders and two receipts and the cargo back to undertaking company. Then one order and one cargo receipt are handed to the keyboard operator; the cargo and one cargo receipt are handed over to treasury manager to put the cargo in storage.

\section{B. Waiting for reception}

The management procedures of waiting for reception are shown in Figure 2. The treasury manager of TPL is responsible foe receiving the cargo delivered by the consignor, and the keyboard operator receives the orders, writes out cargo receipts for delivery staff. The cargo receipts are printed by the system in triplicate. The cargo receipts are delivery receipts in the system, and are printed after the delivery receipts are renamed, in fact this saves handwritten cargo receipts.

The keyboard operator keys the cargo receipt in system to form delivery receipt.

\section{Inbound}

Each branch is equipped with one treasury manager, who is responsible for the inbound and outbound of cargo in kind of the branch.

In the case of door to door service, keyboard operators receive orders and cargo receipts from delivery staff and fill out delivery lists according to cargo receipts as shown in Figure 3.

The form and content of cargo receipts and delivery lists are basically the same. Cargo receipts are handwritten; delivery lists are the electronic edition of cargo receipts.

If the keyboard operators of waiting for reception notify by themselves treasury managers to put cargo in kind in storage, cargo receipts needn't be filled out; delivery lists are written out then printed in triplicate with the name of cargo receipts.

Delivery lists should be filled out whether it is the inbound of cargo in kind that have been stored or the inbound of ordinary cargo.

After the delivery lists are filled out and confirmed, they come into inventory tables and form daily income tables, which are printed by each branch when needed. Numbers of cargo allocation 
in the inventory table are filled out by keyboard operators according to the print form returned by the treasury manager.

The inventory table contains information from delivery lists, including name of shipping enterprise, destination, name of store, name of cargo, declared value, number of packages, weight, volume, cash, account period, arrival pay, back pay, own delivery, other charges, order arrival date, number of orders, number of inventory documents, person handling, alternative payments, the order numbers.

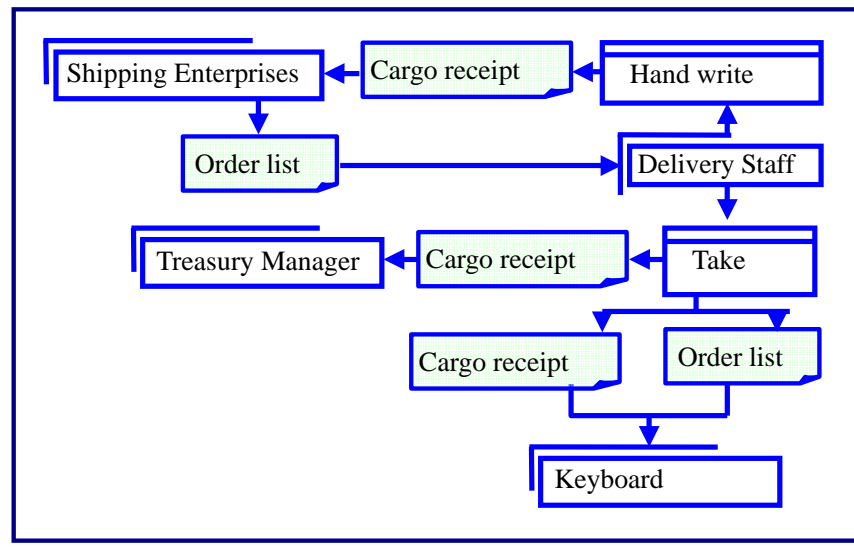

Figure 1. door to door service

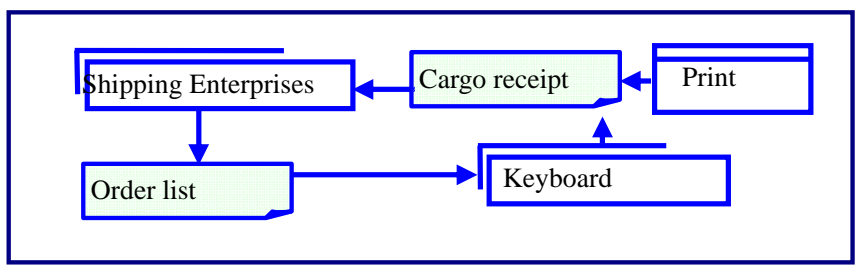

Figure 3. Waiting for reception

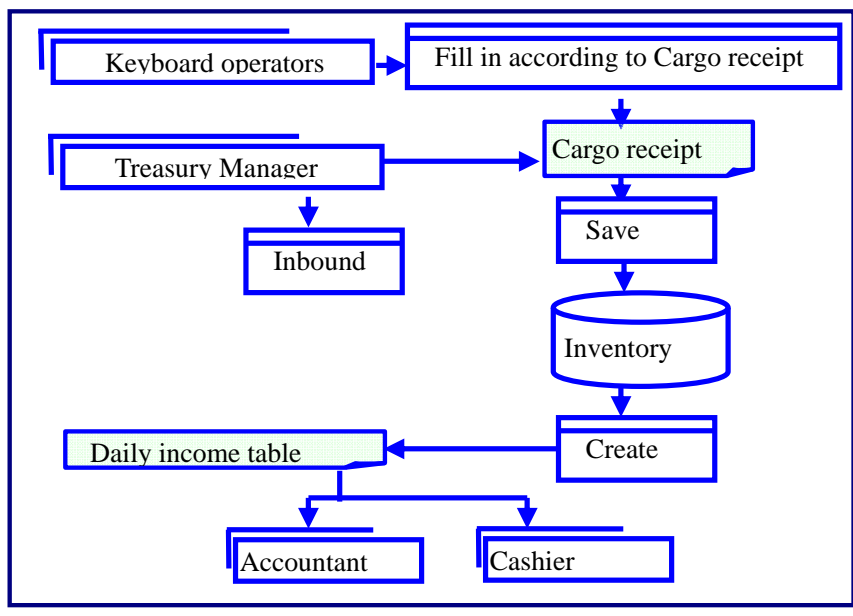

Figure 2. Inbound

The daily income table contains information from delivery lists, including direction, number of packages, volume, weight, cash, account period, arrival pay, total, average piece, number of inventory documents.

For the consignee, as long as there are cargo outbound, whether the cargo are delivered to the destination or not, daily income tables should be formed as it is regarded as getting incomes.

With each inbound, the number on the inventory tables increases, and the quantity of increase should equal that of inbound. The delivery list is the basic form, and on the basis of this delivery list, daily income table, monthly income table, annual income table and inventory table are created.

\section{outbound}

\section{A. Responsibility of outbound}

The keyboard operator is responsible for the outbound of cargo in storage in the branch. He should check the inventory daily, and if the cargo in one direction meet the conditions of delivery (subject to order arrival date, automatically prompted by the system), make outbound in that direction. With each outbound the number in the inventory table decreases, and the quantity change equals the quantity of outbound. In the premise of meeting the order arrival date, heavy argo and light cargo should be outbounded in proportion if possible. The system provides referential notification, which does not carry out compulsorily.

\section{B. Outbound documents}

Be it the outbound of cargo not in storage or in storage, outbound lists should be created and printed, then acceptance sheets are created (corresponding to outbound lists), finally daily settlement tables are created.

For TPL, when cargo are outbound and delivered to destination,, it is seen as tasks completed, settlements can be made and daily settlement tables are formed.

The outbound list contains information from the inventory table, including cargo reception date, 
number of inbound list, name of shipping enterprise, name of store, name of cargo, weight, volume, number of packages, order arrival date, number of orders, number of inventory documents, arrival pay, own delivery, alternative payments, etc. It also contains date of delivery, keyboard operator, storekeeper, distribution staff, person handling, consignee, actual arrival date, and forms the number of outbound list. The outbound list is in duplicate, handed to distribution staff.

The daily settlement table is a complex table, composed of receiving and shipping freight daily settlement table, management fee daily settlement table and enterprise fund balance table.

In the receiving and shipping freight daily settlement table, destination, number of inbound list, name of shipping enterprise, number of packages, receiving freight (average piece, cash, account period, arrival pay, other charges, total) come from the inventory table, kept for settlement. Shipping freights (average piece, cash, account period, total) are kept for settlement. When the amount of account period is paid, the accountant activates and settles the account, and the amount of account period is computed. Income, income rate and alternative payments come form the inventory table. The states of alternative payments are three types, 1. Entrust, 2.Collection, 3.Payment. Default is Entrust. When delivery staff hand alternative payments to the cashier, the cashier hands the receipt to the accountant, who activates this item for the first time, turning it into "collection"; when the shipping enterprise comes for this cargo payment at certain time, the accountant settles the account and the cashier pays it, then the accountant activated this item for the second time, turning it into "payment". The system prompts at each time of activation, and the states cannot be reversed.

\section{Acknowledgements}

Project supported by the Science and Technology Development Program of Shenyang, China (Grant No. F12-169-9-00).

\section{References}

[1]. Juan Ren The development of modern logistics in the information age. Management World. 2005, 11: 152-153.

[2]. Tong Qing-ping, Study on Development Present Situation of TPL in China, Logistics Sci-Tech, 2007. 09

[3]. Wei Qin ,The Exploration of Several Issues on the Foreign Capital Investment in Chinese Logistics Industry , China Market, 2007. 23

[4]. Small and medium sized logistics enterprises in the Information Technologies and Strategies. Federation.of China Logistics and Purchasing. Newsletter2011.1.27-29

[5]. Guidance on the promotion of the work of logistics informationization [Industry and Information Department information [2013] 7]

[6]. Fan Ruguo, Li Yuying. Research on Cost Saving and Benefit Distribution of Logistics System Based on Fourth Party Logistics. Technology Economics.2010.11.127-131.

[7]. Tian Yajuan, Wu Jian, Li Xue, Guan Lili,To explore the 4PL service innovation based on the logistics information platform. The Logistics Engineering and Management .2009.9

[8]. Yan Zhang, Qiong Sun, Yinghong Xie 4PL BUSINESS MODEL DESIGN BASED ON TPL REQUIREMENTS. ICEIS 2011 - Thirteen International Conference on Enterprise Information Systems.702-707

[9]. Yan ZHANG , Yinghong Xie Research into 4PL Management Services Platform Based on Horizontal Supply Chain, ICLEM 2010: Logistics for Sustained Economic Development (c 2010 ASCE,4521-4527.

[10]. Yan Zhang. The design thought based on the practical needs of multi-user 4PL platform . Logistics and Purchasing .2011.6.68-69. 